\title{
Probing the Structure and Dynamics of Molecular Clusters Using Rotational Wave Packets
}

\author{
Gediminas Galinis, ${ }^{1}$ Cephise Cacho, ${ }^{2}$ Richard T. Chapman, ${ }^{2}$ Andrew M. Ellis, ${ }^{3}$ Marius Lewerenz, ${ }^{4}$ Luis G. Mendoza Luna, ${ }^{1}$ \\ Russell S. Minns, ${ }^{5}$ Mirjana Mladenović, ${ }^{4}$ Arnaud Rouzée, ${ }^{6}$ Emma Springate, ${ }^{2}$ I. C. Edmond Turcu, ${ }^{2}$ \\ Mark J. Watkins, ${ }^{1}$ and Klaus von Haeften ${ }^{1, "}$ \\ ${ }^{1}$ University of Leicester, Department of Physics \& Astronomy, Leicester LE1 7RH, United Kingdom \\ ${ }^{2}$ Central Laser Facility, STFC Rutherford Appleton Laboratory, Harwell Oxford, Didcot, Oxfordshire OX11 OQX, United Kingdom \\ ${ }^{3}$ University of Leicester, Department of Chemistry, Leicester LE1 7RH, United Kingdom \\ ${ }^{4}$ Université Paris-Est, Laboratoire Modélisation et Simulation Multi Echelle, MSME UMR 8208 CNRS, \\ 5 bd Descartes, 77454 Marne-la-Vallée, France \\ ${ }^{5}$ University of Southampton, Chemistry, Southampton SO17 1BJ, United Kingdom \\ ${ }^{6}$ Max Born Institute, Max Born Strasse 2A, 12489 Berlin, Germany
}

(Received 26 March 2014; published 24 July 2014)

\begin{abstract}
Rotational wave packets of the weakly bound $\mathrm{C}_{2} \mathrm{H}_{2}-\mathrm{He}$ complex have been created using impulsive alignment. The coherent rotational dynamics were monitored for 600 ps enabling extraction of a frequency spectrum showing multiple rotational energy levels up to $J=4$. spectrum has been combined with $a b$ initio calculations to show that the complex has a highly delocalized structure and is bound only by ca. $7 \mathrm{~cm}^{-1}$. The experiments demonstrate how highly featured rotational spectra can be obtained from an extremely cold environment where only the lowest rotational energy states are initially populated.
\end{abstract}

DOI: 10.1103/PhysRevLett.113.043004

PACS numbers: 33.20.-t, 33.80.-b, 36.40.Mr

Weakly bound molecular complexes are important model systems for condensed matter held together by van der Waals forces [1]. The weak binding leads to large amplitude motion and delocalized structures that are often reflected in complex rotational spectra that cannot easily be categorized through rigid rotor models. Furthermore, obtaining these spectral features imposes practical problems associated with the very low temperatures that are required to form weakly bound complexes. The low temperatures imply the population of only the lowest quantum states. Consequently, the features in conventional rotational (microwave) spectroscopy comprise only a few lines-often not enough for a comprehensive analysis of the structure of the complex [2].

A potential solution to this problem is to use impulsive alignment to generate rotational wave packets through the nonresonant interaction of an intense laser field with a molecule, aligning it in space [3]. Tuning the laser pulse duration and intensity controls the number of rotational eigenstates that contribute to the wave packets. Using this technique for isolated molecules it is possible to monitor the evolution of alignment in time, simultaneously obtaining information on the rotational dynamics and structure [3-10]. The extension of this technique to complex systems where weak interactions are important has been postulated theoretically [11], and demonstrated on the very simplest of systems, noble-gas dimers [12]. The extension of impulsive alignment to van der Waals complexes with internal and delocalized structures remains a challenge. This is due in part to the weak binding and floppy nature of the complex, but also due to the increased complexity of the resulting rotational spectrum, such that currently, to the best of our knowledge, no such measurement has been reported.

This approach may also have potential for exploring liquids, where the wave-packet dynamics will be sensitive to dephasing. A particularly promising liquid to begin such studies is superfluid helium. Frequency-domain spectra of molecules embedded into large superfluid helium droplets show sharp rotational transitions, suggesting that wave packets will not dephase $[2,13]$. However, the recent attempt to impulsively align molecules in large helium droplets by Pentlehner et al. suggests that this may not be the case [14].

This work was motivated to establish a bridge between free molecules and helium droplets. As a starting point we chose to study the complex between acetylene $\left(\mathrm{C}_{2} \mathrm{H}_{2}\right)$ and a single helium atom in order to reduce size and complexity to a minimum. Microwave spectroscopy of $\mathrm{C}_{2} \mathrm{H}_{2}$ has not been performed in large helium droplets nor in small helium complexes, one limitation being a lack of a permanent dipole moment. Several potential energy surfaces (PES) for $\mathrm{C}_{2} \mathrm{H}_{2}-\mathrm{He}$ nevertheless exist, allowing for comparison between experiment and theory [15-17].

A beam comprising $\mathrm{C}_{2} \mathrm{H}_{2}-\mathrm{He}_{n}$ complexes was generated in a pulsed, supersonic expansion using an Even-Lavie nozzle $(d=100 \mu \mathrm{m})$ and propagated through a vacuum apparatus [18]. Expansion conditions were chosen to prevent extensive complex formation and to favor the $n=1$ complex. To excite rotational wave packets $300 \mathrm{fs}$ laser pulses (pump) intersected the beam of complexes followed by 50 fs laser pulses (probe) to detect molecular 


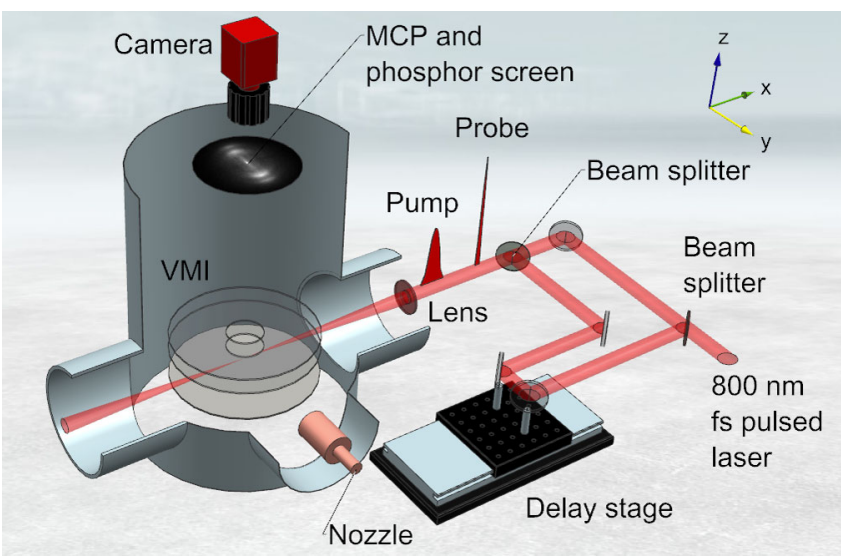

FIG. 1 (color online). Schematic of experimental setup. Helium- $\mathrm{C}_{2} \mathrm{H}_{2}$ complexes produced by expansion of $0.01 \%$ $\mathrm{C}_{2} \mathrm{H}_{2}$ in $9 \mathrm{MPa} \mathrm{He}$ through a cooled Even-Lavie valve were irradiated by femtosecond pulses originating from a $30 \mathrm{fs}, 1 \mathrm{kHz}$ Ti:sapphire laser operating at $800 \mathrm{~nm}$ (KM Labs Red Dragon). Both pump and probe laser beams, comprised of separate grating compressors (not shown), were colinearly focused through a $f=500 \mathrm{~mm}$ lens into the molecular beam $50 \mathrm{~mm}$ downstream from the nozzle exit.

alignment (Fig. 1). Control of the number of rotational levels excited in the wave packets was achieved by variation of the intensity of the pump pulses between $2.5 \times 10^{11}$ and $5 \times 10^{12} \mathrm{~W} \mathrm{~cm}^{-2}$. The probe pulse intensity of $1 \times 10^{15} \mathrm{~W} \mathrm{~cm}^{-2}$ was sufficient to instantly break molecular bonds in a Coulomb explosion, thereby generating $\mathrm{C}^{+}, \mathrm{C}^{2+}$, and $\mathrm{H}^{+}$fragment ions. The velocity vectors of the fragment ions, which carried the molecular alignment information, were selectively detected in a velocity map imaging (VMI) spectrometer [19] whose detector plane was parallel to the polarization plane of both laser beams (xy plane in Fig 1). The two-dimensional projection of the recoiling $\mathrm{C}^{+}$fragment directions and intensities was used to determine $\cos ^{2} \theta$ for each position on the detector, where $\theta$ designates the angle between the polarization of the pump laser and the projected velocity vectors. The average over the entire detector area, $\left\langle\cos ^{2} \theta\right\rangle_{2 \mathrm{D}}$, is proportional to the molecular alignment $[7,14]$. This parameter was measured as a function of time by scanning the delay between pump and probe laser pulses to reveal the rotational dynamics of the complexes.

Figure 2 shows raw Coulomb explosion images for $\mathrm{C}^{+}$ momentum distributions under different expansion conditions. Figure 2(a) shows the ion image under conditions where free $\mathrm{C}_{2} \mathrm{H}_{2}$ (no helium attached) is the dominant molecular species in the expanding gas. This particular image shows relatively sharp features, with evidence of more than one fragmentation channel leading to the formation of $\mathrm{C}^{+}$. The kinetic energies of $\mathrm{C}^{+}$[Fig. 2(c)] are consistent with the previous studies of the strong-laserfield-induced fragmentation of $\mathrm{C}_{2} \mathrm{H}_{2}$ into $\mathrm{C}^{n+}+\mathrm{C}^{m+}$ channels, where $n, m=1,2,3$ [20]. By way of contrast,

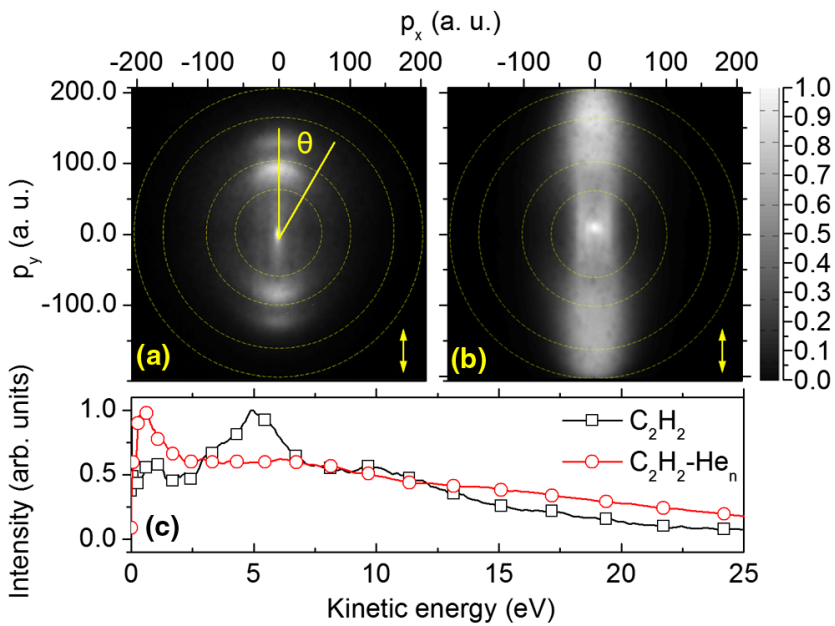

FIG. 2 (color online). Velocity map images of the Coulomb explosion process. These particular images focus on the $\mathrm{C}^{+}$ fragment and were recorded with different nozzle temperatures $T_{0}$, namely, (a) $293 \mathrm{~K}\left(P_{0}=7 \mathrm{MPa}\right)$ and (b) $203 \mathrm{~K}$ $\left(P_{0}=6.4 \mathrm{MPa}\right)$, but under otherwise identical conditions. Corresponding angularly integrated and normalized kinetic energy distributions are shown in (c): pure $\mathrm{C}_{2} \mathrm{H}_{2}$ (black line with squares) and $\mathrm{C}_{2} \mathrm{H}_{2}$ with $\mathrm{C}_{2} \mathrm{H}_{2}-\mathrm{He}_{n}$ complexes present (red line with circles). Production of complexes between helium and acetylene is enhanced in (b), supported by increased kinetic energy compared to (a). The yellow double-headed arrow indicates the direction of the pump and probe laser polarizations, which are parallel to the $y$ axis in Fig. 1. In (a) and (b) the dashed circles are guides to the eye and the axes $p_{x}$ and $p_{y}$ are given in atomic units of momentum.

Fig. 2(b) shows the image obtained when the nozzle was cooled to $203 \mathrm{~K}$ to facilitate the formation of complexes with helium. The angular distribution of the two images is identical, the only difference being higher kinetic energies in image (b). Increased kinetic energy unambiguously indicates the generation of further charges, although the laser parameters themselves have not changed. Spacecharge effects can be excluded given that the temperature variation increases the gas number density in the interaction region only by $30 \%$ - an insignificant reduction in the internuclear separation of ions in the gas compared to the high atomic number density within a complex. We suspect that a mechanism similar to that predicted for large helium droplets doped with $\mathrm{Xe}$ atoms is operating for small complexes [21]. In our case, the ionization probability of $\mathrm{He}$ atoms might be enhanced by the presence of the solvated $\mathrm{C}_{2} \mathrm{H}_{2}$ molecule.

The time-resolved molecular alignment, $\left\langle\cos ^{2} \theta\right\rangle_{2 \mathrm{D}}$, is shown in Fig. 3 for conditions similar to Fig. 2(b). The strongest contribution originates from $\mathrm{C}_{2} \mathrm{H}_{2}$, whose full, half, and quarter revivals reappear every rotational period as maximum alignment and antialignment. The features extend over the full range of the scan up to $600 \mathrm{ps}$ without any appreciable damping in amplitude, so one can infer that the coherence of the rotational wave packet is at least 


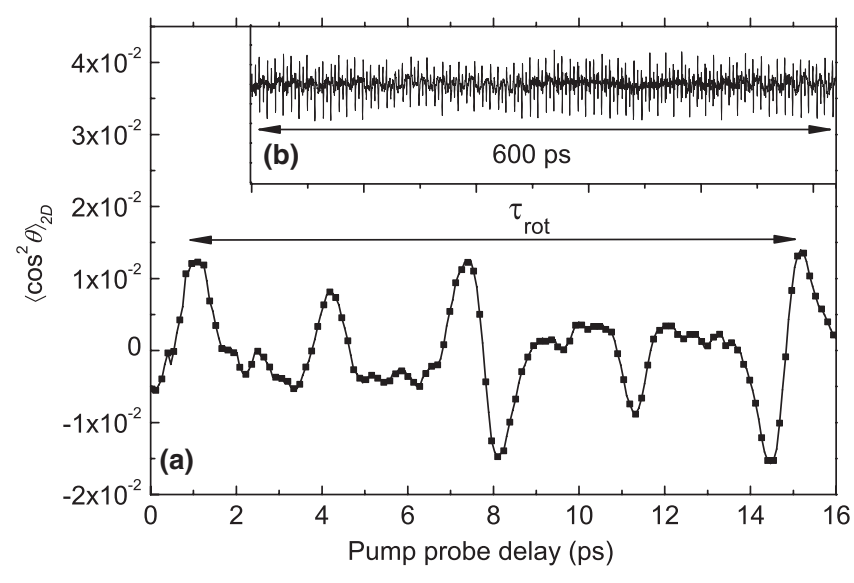

FIG. 3. Time-resolved alignment. (a) Rotational revivals obtained under conditions where the helium stagnation pressure and temperature were $6.4 \mathrm{MPa}$ and $217 \mathrm{~K}$, respectively. Note that the average geometric alignment $\left\langle\cos ^{2} \theta\right\rangle_{\text {geom }}=0.78$ has been subtracted. The data have been smoothed by a five-step-wide moving average filter. The rotational period of acetylene is indicated by a double-headed arrow. (b) For time delays up to the maximum of $600 \mathrm{ps}$, no damping in the amplitude was observed. The coherence time of the wave packet for free $\mathrm{C}_{2} \mathrm{H}_{2}$ molecules is therefore at least 600 ps.

600 ps. Revivals from acetylene-helium complexes $\left(\mathrm{C}_{2} \mathrm{H}_{2}-\mathrm{He}_{n}\right)$ are difficult to directly identify in the alignment scan because of the dominant signals from free (unclustered) acetylene. A much clearer picture is obtained when a Fourier transform is performed, producing a rotational frequency spectrum [22].

Rotational spectra (amplitude spectra) in the frequency domain are shown in Figs. 4(a) and 4(b). A series of discrete lines is seen in the images, which corresponds to particular frequency contributions of $\mathrm{C}_{2} \mathrm{H}_{2}$ to the rotational wave packet. The main features seen in Fig. 4(b) coincide with the beat frequencies of free $\mathrm{C}_{2} \mathrm{H}_{2}$ (no complex with helium) at $6 B+4 n B$, where $B$ is the $\mathrm{C}_{2} \mathrm{H}_{2}$ rotational constant $\left(B=1.1767 \mathrm{~cm}^{-1}\right.$ [23]) and $n=0,1,2,3$, etc. Four members of this series are seen at a pump power of $2.5 \times 10^{11} \mathrm{~W} \mathrm{~cm}^{-2}$. The first line at $6 B$ frequency is due to the $J^{\prime}=2 \leftarrow J^{\prime \prime}=0$ transition, with successive lines at $10 B$, $14 B, 18 B$, etc. The significantly higher intensity of the contribution from the $J=1$ rotational state compared with the $J=0$ state is attributed to nuclear spin statistics [24]. When the pump laser intensity is increased to $5 \times 10^{12} \mathrm{~W} \mathrm{~cm}^{-2}$, higher rotational transitions are induced, reaching up to the $J=13$ rotational energy level.

In the low frequency range, shown in Figs. 5(a), 5(b), and 5(c), several peaks are observed which have frequencies well below the lowest possible rotational transitions of free $\mathrm{C}_{2} \mathrm{H}_{2}$ and are therefore attributed to $\mathrm{C}_{2} \mathrm{H}_{2}-\mathrm{He}_{n}$ complexes. The full widths at half maximum of the $\mathrm{C}_{2} \mathrm{H}_{2}-\mathrm{He}_{n}$ features were found to be $0.03 \mathrm{~cm}^{-1}$, which matches the experimental limit in resolution set by the length of the delay scan of $600 \mathrm{ps}$. Hence, the line width of the peaks assigned

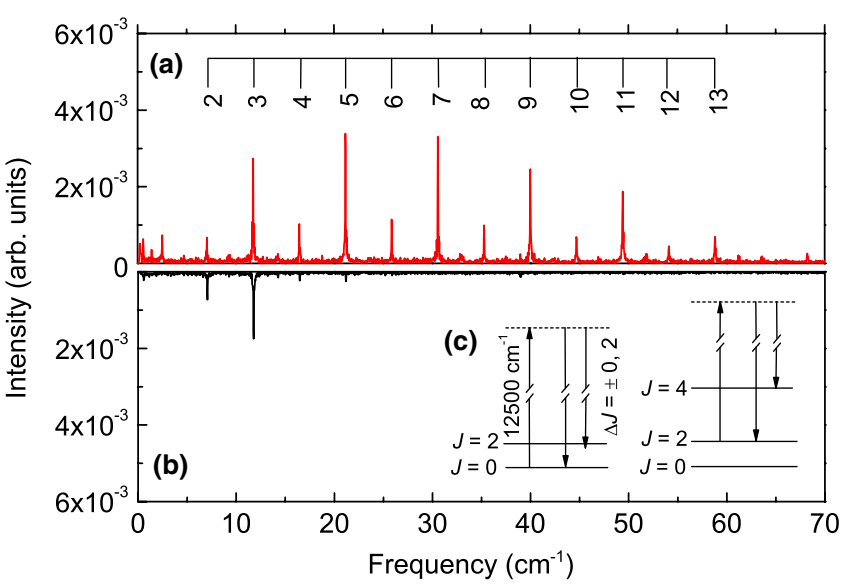

FIG. 4 (color online). Overview spectra. (a) Fourier amplitude spectrum of the time-resolved molecular alignment recorded at a pump laser intensity of $5 \times 10^{12} \mathrm{~W} \mathrm{~cm}^{-2}\left(P_{0}=6.4 \mathrm{MPa}\right.$ and $T_{0}=217 \mathrm{~K}$ ). (b) Spectrum recorded under similar conditions, but at a lower pump laser intensity of $2.5 \times 10^{11} \mathrm{~W} \mathrm{~cm}^{-2}$ $\left(P_{0}=4 \mathrm{MPa}\right.$ and $\left.T_{0}=212 \mathrm{~K}\right)$. The amplitude spectra are dominated by strong peaks arising from beats between rotational levels of free $\mathrm{C}_{2} \mathrm{H}_{2}$ connected by $\Delta J= \pm 2$. The labels of the transitions correspond to the $J$ quantum number of the final states, e.g., " 2 " labels the final state of the $J^{\prime}=2 \leftarrow J^{\prime \prime}=0$ excitation. (c) The rotational levels in the wave packet are excited via virtual states through sequential Raman excitations with an $800 \mathrm{~nm}\left(12500 \mathrm{~cm}^{-1}\right)$ laser pulse.

to $\mathrm{C}_{2} \mathrm{H}_{2}-\mathrm{He}_{n}$ complexes is consistent with a coherence time for the rotational wave packet of at least $600 \mathrm{ps}$. This first observation of coherent propagation of rotational wave packets in small $\mathrm{C}_{2} \mathrm{H}_{2}-\mathrm{He}_{n}$ complexes contrasts with the strong dephasing found in large helium droplets [14]. As detailed below, the strongest features in each of the spectra can be assigned to the $\mathrm{C}_{2} \mathrm{H}_{2}-\mathrm{He}$ complex, which will be the focus of the remainder of the Letter. Other weaker and unlabeled features are attributed to larger complexes with more than one helium atom attached: further discussion of these is held over to a future publication.

To guide the assignment, the interaction energies for the $\mathrm{C}_{2} \mathrm{H}_{2}-\mathrm{He}$ complex were calculated over a wide range of geometries using the coupled cluster singles and doubles with perturbative triples [CCSD(T)] method [25-27]. An analytical representation of the PES at the infinite basis limit was used to predict rovibrational levels and wave functions. A detailed description of these calculations will be given in a forthcoming publication [28]. Selected levels and transition energies from these calculations are displayed in Fig. 6. The computed rovibrational ground state energy is $-7.417 \mathrm{~cm}^{-1}$ relative to the dissociation limit into $\mathrm{C}_{2} \mathrm{H}_{2}+\mathrm{He}$. Only the total angular momentum quantum number $J$ and the parity of the wave functions are rigorous quantum numbers. Parity is coded by the symbols $e$ and $f$ for levels with parity $+(-1)^{J}$ and $-(-1)^{J}$, respectively. We can introduce another quantum number $j$, which refers to the internal rotation of the $\mathrm{C}_{2} \mathrm{H}_{2}$ unit. Although 


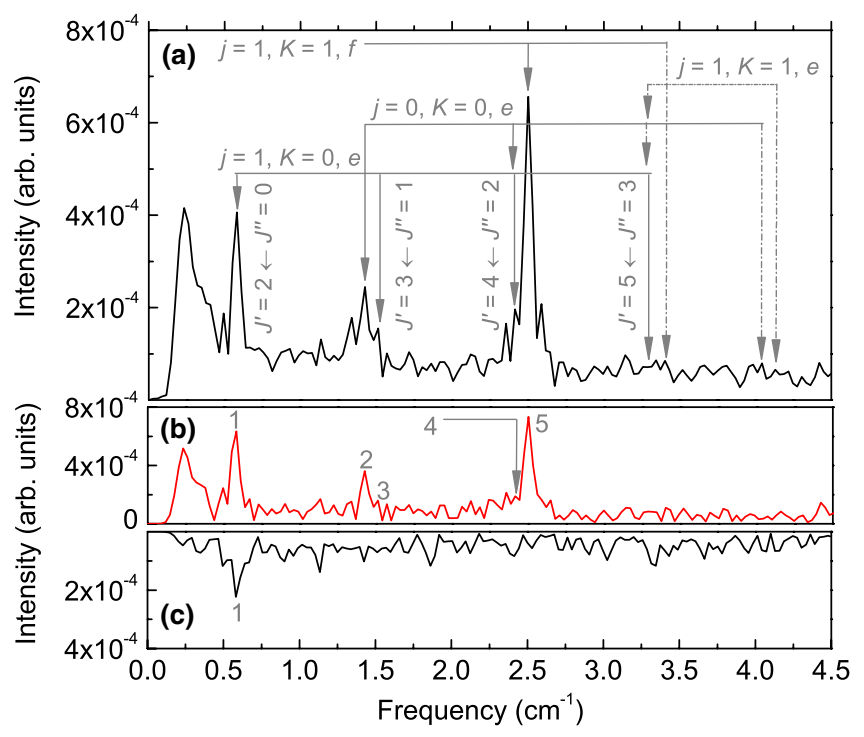

FIG. 5 (color online). Expanded view of the frequency region below $5 \mathrm{~cm}^{-1}$. An average of five spectra recorded for similar expansion conditions is shown in panel (a). Two spectra acquired at pump laser intensities of $5 \times 10^{12}$ and $2.5 \times 10^{11} \mathrm{~W} \mathrm{~cm}^{-2}$ are shown in panels (b) and (c), respectively. Prominent features assigned to $\mathrm{C}_{2} \mathrm{H}_{2}-\mathrm{He}$ are also annotated by numbers $1-5$. The transitions which were not safely established in (a) are marked by dashed-dot arrows. See text and the energy level diagram in Fig. 6 for further information. Amongst other unassigned features, the peak at $0.25 \mathrm{~cm}^{-1}$ is particularly prominent. We note that larger complexes may contribute lines and that this low frequency region is particularly susceptible to artifacts, hence we treat this region with caution.

approximate, this turns out to be a useful quantum number, which is consistent with the floppy character of the binary complex. The states are additionally labelled by the approximate quantum number $K$ for the projection of $J$ onto the body-fixed $z$ axis, chosen to be aligned with the intermolecular (Jacobi) vector in our calculations.

Inspection of the energy level structure and analysis of the wave-function properties reveal that the $\mathrm{C}_{2} \mathrm{H}_{2}-\mathrm{He}$ complex is a highly delocalized system which does not exhibit the typical rotational pattern of a linear molecule in spite of its linear minimum energy structure. In the linearmolecule model, the rotational excitation of the vibrational ground state of $\mathrm{C}_{2} \mathrm{H}_{2}-\mathrm{He}$ is characterized by $B_{0}=$ $0.245 \mathrm{~cm}^{-1}$ and a large centrifugal distortion constant $D_{0}=3 \times 10^{-4} \mathrm{~cm}^{-1}$. In the bent-molecule model, we derived $A_{0}=3.29 \mathrm{~cm}^{-1}, \bar{B}_{0}=\left(B_{0}+C_{0}\right) / 2=0.24 \mathrm{~cm}^{-1}$, and a large $\Delta_{0}=B_{0}-C_{0}$ value of $0.37 \mathrm{~cm}^{-1}$ from the $J=1$ results. A prominent rotational perturbation, seen in both model descriptions, is due to a strong vibrationrotation (Coriolis) coupling. The latter effect is also evident in a large separation of $0.37 \mathrm{~cm}^{-1}$ between the $(1,1)^{e}$ and $(1,1)^{f}$ levels in Fig. 6. The rotational dynamics of $\mathrm{C}_{2} \mathrm{H}_{2}-\mathrm{He}$ is, thus, different from the corresponding low-energy rotational behaviour of the isolated $\mathrm{C}_{2} \mathrm{H}_{2}$

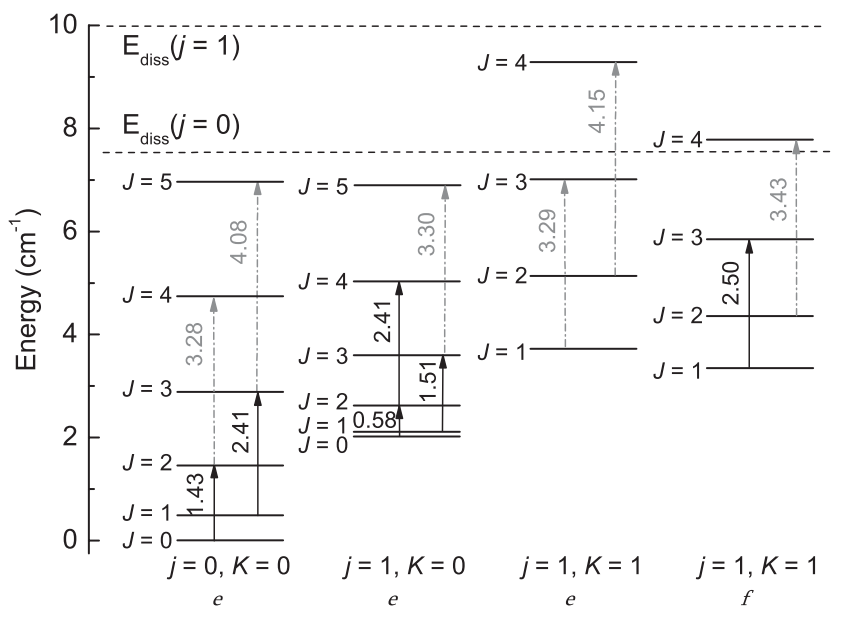

FIG. 6. Rotational energy level diagram of $\mathrm{C}_{2} \mathrm{H}_{2}-\mathrm{He}$. The arrows designate the transitions observed, whereas the dasheddotted, gray arrows indicate transitions which were not safely established in the experimental data.

molecule, which is characterized by $D_{0}\left(\mathrm{C}_{2} \mathrm{H}_{2}\right)=1.51 \times$ $10^{-6} \mathrm{~cm}^{-1}$ [24] and a small $l$-type doubling constant $q \approx 0.006 \mathrm{~cm}^{-1}$ [29].

Based on these results, six lines were assigned to $\mathrm{C}_{2} \mathrm{H}_{2}-\mathrm{He}$, as shown in Fig. 5(a), yielding an agreement between theory and experiment within $0.03 \mathrm{~cm}^{-1}$. We note that the new PES provides a better match to the measured line positions than the best PES available prior to this work [17]. The expected transitions for $\mathrm{C}_{2} \mathrm{H}_{2}-\mathrm{He}$ are marked in Fig. 6 by vertical arrows and text, showing the energy differences in $\mathrm{cm}^{-1}$. The assignment of the lines in the low frequency range is also shown in Table $\mathrm{I}$ in the Supplemental Material [30].

The identified $\mathrm{C}_{2} \mathrm{H}_{2}-\mathrm{He}$ features depend distinctly on the pump laser intensity, as shown in Figs. 5(b) and 5(c). At the lowest pump laser intensity employed, shown in Fig. 5(c), only the $J^{\prime}=2 \leftarrow J^{\prime \prime}=0$ transition in the $j=0, K=0, e$ manifold can be reliably assigned. However, the intensities of other lines grow with increasing pump laser energy, with marked changes in the relative intensities. The impact of the pump laser intensity on the relative contributions of specific beat frequencies to the rotational wave packet will be explored in a future publication.

In summary, using impulsive alignment we have created coherent rotational wave packets in the weakly bound $\mathrm{C}_{2} \mathrm{H}_{2}-\mathrm{He}$ complex. The dynamics of this wave packet were followed in the time domain for $600 \mathrm{ps}$, allowing us to derive a frequency spectrum containing multiple rotational energy levels up to $J=4$, covering more rotational states than could be achieved through conventional rotational spectroscopy. The spectrum provides the first experimental determination of the rotational energy levels which, when combined with state of the art $a b$ initio calculations, confirms a highly delocalized structure for $\mathrm{C}_{2} \mathrm{H}_{2}-\mathrm{He}$. The experiments therefore not only provide the first 
experimental measurements on this particular complex, they also demonstrate more generally that it is possible to use impulsive alignment to investigate rotational levels in exceptionally weakly bound complexes. This work opens the way for the study of size-dependent effects in condensed matter physics using impulsive alignment, such as incipient superfluidity in molecule-doped helium clusters.

The authors wish to thank STFC for access to the Artemis facility at the Rutherford Appleton Laboratory and the University of Leicester for funding to support the studentship for G. G. We would like to thank S. Hook, P. Rice, N. Rodrigues, and S. Thornton for technical support during the experiment, as well as J. Underwood and M. Siano for their contributions to the design of the VMI spectrometer. $\mathrm{KvH}$ kindly acknowledges funding by STFC (seed corn fund for experiments using 4th generation light sources) and the Leverhulme Trust (F/00212/AH). L. G. M. L. acknowledges financial support from the Mexican Consejo Nacional de Ciencia y Tecnología (CONACYT) Scholarship No. 310668, ID 215334. R. S. M. would like to thank the Royal Society for support (UF100047). M. L. and M. M. acknowledge computational resources funded through ANR Grant No. ANR-08BLAN-0146-01.

*kvh6@le.ac.uk

[1] Z. Bačić and R. E. Miller, J. Phys. Chem. 100, 12945 (1996).

[2] L. A. Surin, A. V. Potapov, B. S. Dumesh, S. Schlemmer, Y. Xu, P. L. Raston, and W. Jäger, Phys. Rev. Lett. 101, 233401 (2008).

[3] T. Seideman, Phys. Rev. Lett. 83, 4971 (1999).

[4] F. Rosca-Pruna and M. J. J. Vrakking, Phys. Rev. Lett. 87, 153902 (2001).

[5] J. Itatani, J. Levesque, D. Zeidler, H. Niikura, H. Pépin, J.-C. Kieffer, P. B. Corkum, and D. M. Villeneuve, Nature (London) 432, 867 (2004).

[6] T. Seideman and E. Hamilton, Adv. At. Mol. Opt. Phys. 52, 289 (2005).

[7] O. Ghafur, A. Rouzée, A. Gijsbertsen, W. K. Siu, S. Stolte, and M. J. J. Vrakking, Nat. Phys. 5, 289 (2009).

[8] C. Z. Bisgaard, O. J. Clarkin, G. Wu, A. M. D. Lee, O. Geßner, C. C. Hayden, and A. Stolow, Science 323, 1464 (2009).
[9] T. Kanai, S. Minemoto, and H. Sakai, Nature (London) 435, 470 (2005).

[10] R. Velotta, N. Hay, M. B. Mason, M. Castillejo, and J. P. Marangos, Phys. Rev. Lett. 87, 183901 (2001).

[11] S. Ramakrishna and T. Seideman, Phys. Rev. Lett. 95, 113001 (2005).

[12] J. Wu, A. Vredenborg, B. Ulrich, L. P. H. Schmidt, M. Meckel, S. Voss, H. Sann, H. Kim, T. Jahnke, and R. Dörner, Phys. Rev. A 83, 061403 (2011).

[13] M. Hartmann, R. E. Miller, J. P. Toennies, and A. F. Vilesov, Phys. Rev. Lett. 75, 1566 (1995).

[14] D. Pentlehner, J. H. Nielsen, A. Slenczka, K. Mølmer, and H. Stapelfeldt, Phys. Rev. Lett. 110, 093002 (2013).

[15] R. Moszynski, P. E. S. Wormer, and A. van der Avoird, J. Chem. Phys. 102, 8385 (1995).

[16] M. Rezaei, N. Moazzen-Ahmadi, A. R. W. McKellar, B. Fernández, and D. Farrelly, Mol. Phys. 110, 2743 (2012).

[17] B. Fernández, C. Henriksen, and D. Farrelly, Mol. Phys. 111, 1173 (2013).

[18] U. Even, J. Jortner, D. Noy, N. Lavie, and C. CossartMagos, J. Chem. Phys. 112, 8068 (2000).

[19] A. T. J. B. Eppink and D. H. Parker, Rev. Sci. Instrum. 68, 3477 (1997).

[20] C. Cornaggia, D. Normand, and J. Morellec, J. Phys. B 25, L415 (1992).

[21] A. Mikaberidze, U. Saalmann, and J. M. Rost, Phys. Rev. Lett. 102, 128102 (2009).

[22] A. Przystawik et al., Phys. Rev. A 85, 052503 (2012).

[23] M. Herman, A. Campargue, M. I. El Idrissi, and J. Van der Auwera, J. Phys. Chem. Ref. Data 32, 921 (2003).

[24] G. Herzberg, Molecular Spectra \& Molecular Structure Vol. II, Infrared and Raman Spectra of Polyatomic Molecules (Krieger, Malabar FL, 1991).

[25] C. Hampel, K. A. Peterson, and H.-J. Werner, Chem. Phys. Lett. 190, 1 (1992).

[26] M. J. O. Deegan and P. J. Knowles, Chem. Phys. Lett. 227, 321 (1994).

[27] P. J. Knowles, C. Hampel, and H. J. Werner, J. Chem. Phys. 99, 5219 (1993).

[28] G. Galinis et al., Faraday Discuss., doi:10.1039/c4fd00099d (2014).

[29] G. Herzberg, Molecular Spectra \& Molecular Structure Vol. III, Electronic Spectra and Electronic Structure of Polyatomic Molecules (Krieger, Malabar FL, 1991).

[30] See Supplemental Material at http://link.aps.org/ supplemental/10.1103/PhysRevLett.113.043004 for a table of rotational Raman transitions of $\mathrm{C}_{2} \mathrm{H}_{2}-\mathrm{He}$. 